\title{
Exo-zodiacal disk mapper: a space interferometer to detect and map zodiacal disks around nearby stars
}

Pierre Y. Bely, Richard Burg, Larry Petro, Jean Gay, Pierre Baudoz, et al.

Pierre Y. Bely, Richard Burg, Larry Petro, Jean Gay, Pierre Baudoz, Yves Rabbia, Jean-Marie Perrin, Lawrence A. Wade, Charles A. Beichman, "Exozodiacal disk mapper: a space interferometer to detect and map zodiacal disks around nearby stars," Proc. SPIE 3350, Astronomical Interferometry, (24 July 1998); doi: 10.1117/12.317135

EDIE Event: Astronomical Telescopes and Instrumentation, 1998, Kona, HI, United States 


\title{
The Exo-Zodiacal Disk Mapper: a Space Interferometer to Detect and Map Zodiacal Disks around Nearby Stars
}

\author{
Pierre Y. Bély ${ }^{a}$, Richard Burg $^{b}$, Larry Petro $^{a}$, Jean Gay $^{c}$, Pierre Baudoz $^{c}$, Yves Rabbia $^{c}$, \\ Jean-Marie Perrin ${ }^{d}$, Lawrence Wade ${ }^{e}$, and Charles C. Beichman ${ }^{e}$ \\ ${ }^{a}$ Space Telescope Science Institute, 3700 San Martin Drive, Baltimore, MD 21218 \\ ${ }^{b}$ Johns Hopkins University, Baltimore, Md, 21218 \\ ${ }^{c}$ Observatoire de la Côte d'Azur, Caussols, France \\ ${ }^{d}$ Observatoire de Haute Provence, St. Michel, France \\ e Jet Propulsion Laboratory, Pasadena, Ca, 91109
}

\begin{abstract}
We propose a concept for a space mission designed to make a survey of potential zodiacal dust disks around nearby stars in the mid-IR. We show that a 10-meter baseline nulling interferometer with two 0.6-meter apertures located in a $1 \times 4 \mathrm{AU}$ heliocentric orbit would allow for the survey of about 400 stars in the solar neighborhood and permit a first order determination of the disk inclination and of the dust density and temperature radius dependence. The high dynamic range of the instrument may also be used to study a additional astrophysical phenomena. Beyond its own scientific merit, such a mission would also serve as a technological precursor to a larger interferometer of the type being considered for the detection of earth-like planets.
\end{abstract}

Keywords: Circumstellar disks, zodiacal dust disks, space telescopes, coronagraph

\section{INTRODUCTION}

The direct detection and characterization of earth-like planets is one of the main goals of the NASA Origins program. A keystone mission of that program, the "Terrestrial Planet Finder" or TPF, is a large nulling interferometer which in its current design consists of four 1.5 meter telescopes on a 70-100 meter baseline ${ }^{1}$. However, a number of scientific and technical achievements must be accomplished before such a major instrument can be built. Among the most significant scientific questions that need to be answered is the existence, intensity and orientation of potential zodiacal dust disks in candidate extra-solar systems.

The luminosity of the dust in the inner $10 \mathrm{AU}$ of the Solar System is of order $10^{-7} L_{\odot}$, whereas the luminosity of Earth is $5 \times 10^{-10} L_{\odot}$. In the infrared, at $10 \mu$ mthe flux of the Zodiacal Dust is $F_{Z D} \simeq 1 \times 10^{-4} F \odot$ and the flux of Earth is $F_{\text {Earth }}=5 \times 10^{-7} F_{\odot}$. Therefore, whether in the visible or in the infrared, methods for the detection of extrasolar planets have to contend not only with the glare of the adjacent star but also with that of the enveloping zodiacal dust. Nulling interferometers efficiently cancel the flux of the adjacent star. However, as the planets are not resolved, a substantial zodiacal dust signal is still detected. This zodiacal dust signal, as suggested by the Solar System example, may be orders of magnitude brighter than terrestrial planets. Detection of an earth-like planet will become difficult if the exo-zodiacal disk is much brighter than our own, or close to edge on.

This is a critical issue because of the small number of stars that a given mission can investigate. The first restriction comes from the limited mission duration and sky coverage. The integration times required are fairly long and the number of stars that can be observed during a mission's lifetime is not generous. This is exacerbated by the incomplete sky coverage due to sun constraint on the interferometer: because of the elliptic heliocentric orbit proposed for TPF, more than one third of the sky will not be accessible.

A related problem arises from the fixed baseline of the interferometer. Maximum response from a potential planet occurs when its angular separation from the nulled parent star is such that it is at a peak of the fringe pattern. For detection purposes, this can be adjusted to some extent by changing the wavelength. For spectroscopic analysis, however, an interferometer with a given baseline will be blind to certain discrete angular separations. 
A consequence of these limitations is that the success of the TPF critically depends upon tuning the interferometer design and the observing program to a set of preselected candidate stars. It will therefore be critical to survey a large sample of stars for the presence, orientation, gross structure and brightness of zodiacal dust disks.

In this paper we review what is known about zodiacal disks, examine how current or planned facilities could be used to detect them and propose a space observatory dedicated to the detection and mapping of potential extra-solar zodiacal disks around neighboring stars.

\section{DETECTING DISKS WITH EXISTING OR PLANNED FACILITIES}

Direct detection of exo-zodiacal disks may be attempted with ISO or SIRTF, but neither of these facilities has sufficient spatial resolution or sensitivity for detailed characterization. Obviously, the disks cannot be resolved: a circumstellar disk similar to ours at 10 parsecs would be about 0.6 arcseconds in diameter, as compared to an Airy disk diameter of 6 arcseconds at 10 microns for SIRTF. The sensitivity of these facilities is also insufficient. At 20 microns, where the flux density contrast and photon count combination is the most favorable, the integrated flux of a zodiacal disk similar to ours is $3 \cdot 10^{-4}$ the flux of the sun in a $20 \%$ passband. To detect disks no brighter than 5 times that value would require measuring an IR excess at the $10^{-3}$ level, which is very unlikely because of the uncertainty in the stellar luminosity distribution.

Another possibility would be to work in the visible using a coronagraphic instrument on HST. At 3 to 4 Airy rings from the star, the practical limit for a coronagraph (about 0.2 arcseconds in HST's case), the star's attenuation is about $10^{-5}$, while the intensity of a zodiacal disk similar to ours at 10 parsecs would be about $10^{-8}$ of the parent star. Measuring such a dynamic $(0.1 \%)$ range is very difficult. But a disk edge-on and with 5 times the intensity of our zodiacal disk might be detectable. A survey of stars up to 10 parsecs away would certainly bring very valuable information but would not provide a sufficient sample. Edge-on disks are also the least attractive candidates for a planet finding mission.

Finally, some preliminary detection and crude mapping could be attempted from the ground using large interferometers such as the planned twin Keck or the ESO VLTI. Using simulation techniques described later we have made a detailed calculation of the performance obtainable from using two 10-meter telescopes in an interferometric mode with an 85 meter baseline at 10 microns. Such an interferometer may be useful to detect high-intensity zodiacal clouds. But to reach a flux level comparable to our solar system zodiacal disk with 3- $\sigma$ detection, the survey would be limited to about 20 stars within 10 parsecs. Moreover, because of atmospheric wavelength limitation and the inability to fully rotate the interferometer, the detailed analysis of the disk inclination, structure and temperature distribution of the disk is impossible.

In conclusion, observations with existing facilities might lead to a better understanding of existence of zodiacal disks in about 20 neighboring stars, but this is not a sufficient sample to allow the proper design of a mission of the size of the TPF program. Clearly, a new space instrument is needed for the detailed study of zodiacal disks over a sample large enough to be significant.

A promising approach would be to use the currently studied 4-8 meter Next Generation Space Telescope (NGST) which is planned for launch in 2007 or later $^{2}$. Equipped with an interferometric coronagraphic camera of the type described later in this paper, and working around 10 microns, an 8 meter space telescope would measure zodiacal disks around some 400 stars out to 20 parsecs $^{3}$.

Short of instrumenting NGST for that specific purpose, or if an earlier determination is needed for designing the proposed planet finder mission, a dedicated space instrument would be needed. This is the programmatic justification for the mid-sized space mission that we are proposing, a mission that we call the Exo-Zodiacal Mapper (EZM).

\section{PROPOSED CONCEPT}

Our proposed concept is a nulling two-aperture interferometer of the type first proposed by Bracewell ${ }^{4,5}$ in which the wavefronts from the two apertures are combined out of phase to null out the on-axis star. Off-axis sources such as potential planets or zodiacal disks can be detected by rotating the instrument around its optical axis in order to modulate their incoming signals over the interferometer response pattern. Observations are done in the near infrared, between 7 and $13 \mu \mathrm{m}$. The exact range is set by practical implementation considerations: the upper limit is fixed by the minimal resolution needed with a given baseline and the lower limit is due to the technical feasibility of the super smooth optics required for very high star/zodiacal dust contrast observations. 
We have found that the minimum configuration for a spacecraft at $1 \mathrm{AU}$ would be a baseline of 20 meters and two apertures of 1.5 meters in diameter. However, the size of the instrument can be reduced significantly by locating it farther from the sun, as proposed for TPF. At $3 \mathrm{AU}$ from the sun, where our zodiacal light is reduced by about 2 orders of magnitude, a baseline of 10 meter and mirrors of 0.6 meter in diameter is sufficient, and this is what we have selected for our proposed concept. These parameters have been determined using the computer simulation described in the following section.

The heart of the system is a beam combiner which must be achromatic in order to create a star null over the wide bandpass necessary for high sensitivity. Hence the $180^{\circ}$ phase shift required for the on-axis cancellation must not by obtained by changing the path in one arm by $\lambda / 2$, which would only be true for monochromatic light, but by flipping the polarization vector. The beam combiner which we propose is based on a novel design developed at the Observatoire de la Côte $\mathrm{d}^{\prime} \mathrm{Azur}^{6}$ and named an interferometric coronagraph. In a traditional rotation shearing interferometer ${ }^{7,8,9}$ the $180^{\circ}$ rotation of the pupil is achieved by modifying the traditional Michaelson interferometer with roof prisms (or dihedral mirrors) to flip the beams and with one arm of the interferometer constructed out of the plane. This allows the $S$-component of one beam and the $P$-component of the other beam to be rotated by the required $180^{\circ}$ while keeping the complementary component of each beam in their original states. This approach is simple in that only flat mirrors are necessary but it has two drawbacks. First, since each reflection is $45^{\circ}$ and the $S$ and $P$ waves are not reflected in a symmetric manner polarization-dependent attenuation and anomalous phase delays can cause the beams to become imbalanced. Second, the constructive interference exit port coincides with the entrance port and is thus inaccessible. The design proposed by Gay and Rabbia is a modification of the Mach-Zender interferometer. All optical paths lie in the plane. One of the interferometer arms uses only reflections on flat mirrors, while the other uses a "cat's eye", a two mirror system which functions as a unit magnification relay and has an intermediary focus at the secondary mirror. The (achromatic) $180^{\circ}$ phase shift in this arm occurs as the rays pass through focus ${ }^{10,11}$. All angles are kept small, $S$ and $P$ components are reflected in a symmetric manner, and both the constructive and destructive ports are accessible, the constructive port being useful for monitoring and controlling optical path differences. This optical design uses the beamsplitters in a symmetrical way. The light from each telescope uses the beamsplitter once in transmission and once in reflection. This gets around the problem of fabricating a beamsplitter which is exactly $50 \% / 50 \%$ over a large bandpass. The nulling combiner proposed by Gay and Rabbia has been proven in the laboratory and is presently undergoing testing on a telescope at the Observatoire de la Côte d'Azur. A cancellation level to a few percent has already been achieved and further improvements are expected. It must be noted in this respect that the nulling requirement for exo-zodiacal disk detection is two orders of magnitude less stringent than it would have to be for planet detection $\left(10^{-4}\right.$ instead of $\left.10^{-6}\right)$.

The interferometer optical system is shown schematically in Fig. 1 and an artist's view of the spacecraft concept is shown in Fig. 2. The instrument is composed of two telescopes at the extremities of a boom. The central hub contains the beam combiner, the science instruments and the space support systems. A sun shield protects the interferometer and supports solar arrays for power generation. The two telescopes are of the off-axis type to provide a clean pupil and simplify stops and spatial filters. Each is a classical Cassegrain system to which is added a third powered mirror to provide an accessible pupil where a fast steering mirror can be located. The spacecraft is on an elliptical heliocentric orbit with an aphelion of about 4 astronomical units. The instrument is restricted from pointing more than about $45^{\circ}$ from the anti-sun-earth line direction in order to always keep the telescopes in the shade. The telescopes are passively cooled to about 50 Kelvin to render their thermal emission negligible. The detectors are $\mathrm{Si}$ :As Impurity Band Conduction (IBC), cryogenically cooled to $6 \mathrm{~K}$ to minimize their dark current.

The spacecraft is rotating around the hub axis at the rate of about one turn per hour. Typical observations for a given star last from one hour to a day depending on heliocentric distance, after which the instrument is pointed to the next program star in a nearby direction. In a year, this allows the observation of the candidate stars in a \pm $45^{\circ}$ ecliptic latitude band.

The supporting structure and the sunshield are made of a deployable system, either mechanical or inflatable.

\section{IMAGE RECONSTRUCTION SIMULATIONS}

\subsection{Model}

We have a developed a computer program to determine the signal to noise ratio required for a faithful reconstruction of a zodiacal disk geometry, dust density and temperature. The disks are modeled as geometrically thin disks with 


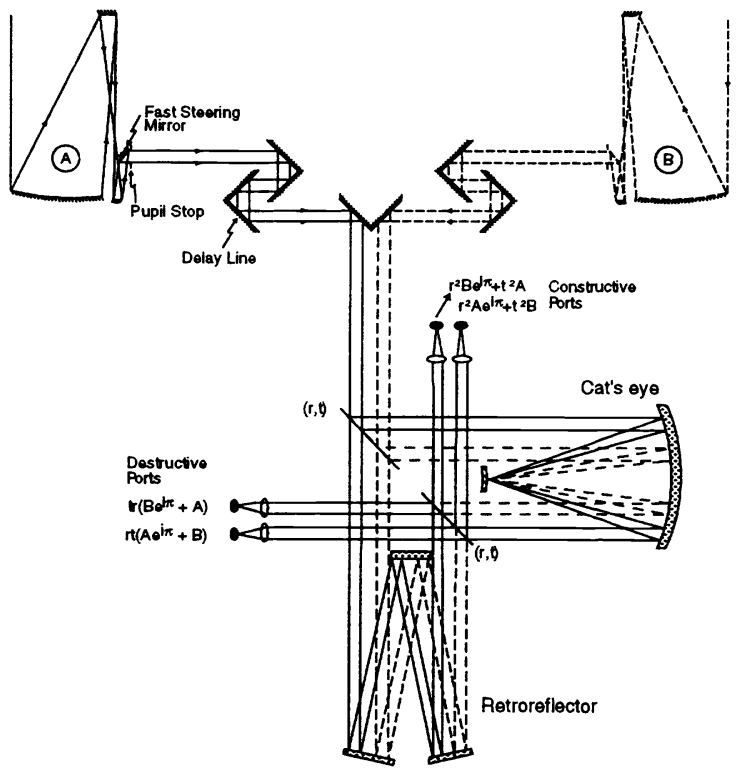

Figure 1. Schematic optical diagram of the nulling interferometer with the two telescopes at the top and the beam combiner below (drawing not to scale). There are two sets of exit ports, one constructive, the other destructive. Beams from the two telescopes which exit the constructive port are asymmetric to each other with respect to the number of reflections and transmissions through the beamsplitter. Since the reflected and transmitted beams are $90^{\circ}$ out of phase, this results in an additional $180^{\circ}$ phase shift between beams from the two telescopes which cancels out the $180^{\circ}$ phase difference between the two arms (to within the equality of the beamsplitter transmission and reflection coefficients). Beams that exit the destructive port are completely symmetric with respect to the number of reflections and transmissions at the beamsplitter, and the only phase difference is due to the $180^{\circ}$ phase shift at the the cat's eye. This results in a null which is fully achromatic and does not depend on the exact reflection/transmission characteristics of the beamsplitter.

a density and temperature varying as power laws. The resulting black body emission by an area $d \sigma$ in a solid angle $d \omega$ is of the form

$$
E=\frac{A e_{o} d \omega d \sigma\left(\frac{r_{o}}{r}\right)^{m}}{\cos \alpha} \lambda \Delta \lambda c e^{\frac{h c}{k \lambda T_{o}\left(\frac{x_{o}}{r}\right)^{n}}}
$$

where $A$ is an intensity scaling constant, $r$ the distance from the parent star, $r_{o}$ the distance at which the equilibrium temperature of the disk material is the same as that near earth (1 AU for a G2 star), $T_{o}$, the temperature (266 K), $e_{o}$ is a reference emissivity equal to that of our zodiacal light at the distance $r_{o}\left(e_{o}=1.12610^{-7}\right.$, i.e. twice the emissivity of our zodiacal light as seen from the earth in a direction perpendicular to the ecliptic), $\lambda$ is the wavelength, $\Delta \lambda$ the bandpass, $h, k, c$ the Planck constant, Boltzman constant and speed of light respectively, $\alpha$ the disk inclination with respect to the line of sight, and $m$ and $n$ are two free parameters. Using the model developed by $\mathrm{J}$. Good ${ }^{12}$, the parameters for our solar system zodiacal disk are: $A=1, m=0.8$ and $n=0.36$.

The response of the nulling interferometer at a field angle $\theta$ and azimuth angle $\phi$ on the sky is:

$$
\mathcal{R}=\frac{1}{2}\left(\cos \left(\frac{\pi D \theta \sin \phi}{\lambda}-\frac{\pi}{4}\right)-\cos \left(\frac{\pi D \theta \sin \phi}{\lambda}+\frac{\pi}{4}\right)\right)^{2}
$$

where $D$ is the baseline separating the two apertures and $\lambda$ the wavelength. The response function is a series of parallel fringes in the direction perpendicular to the baseline and with an angular spacing of $\frac{\lambda}{D}$, or 52 milliarcseconds at $10 \mu \mathrm{mfor}$ a baseline of $10 \mathrm{~m}$. A typical response function is shown in Fig. 3 . 


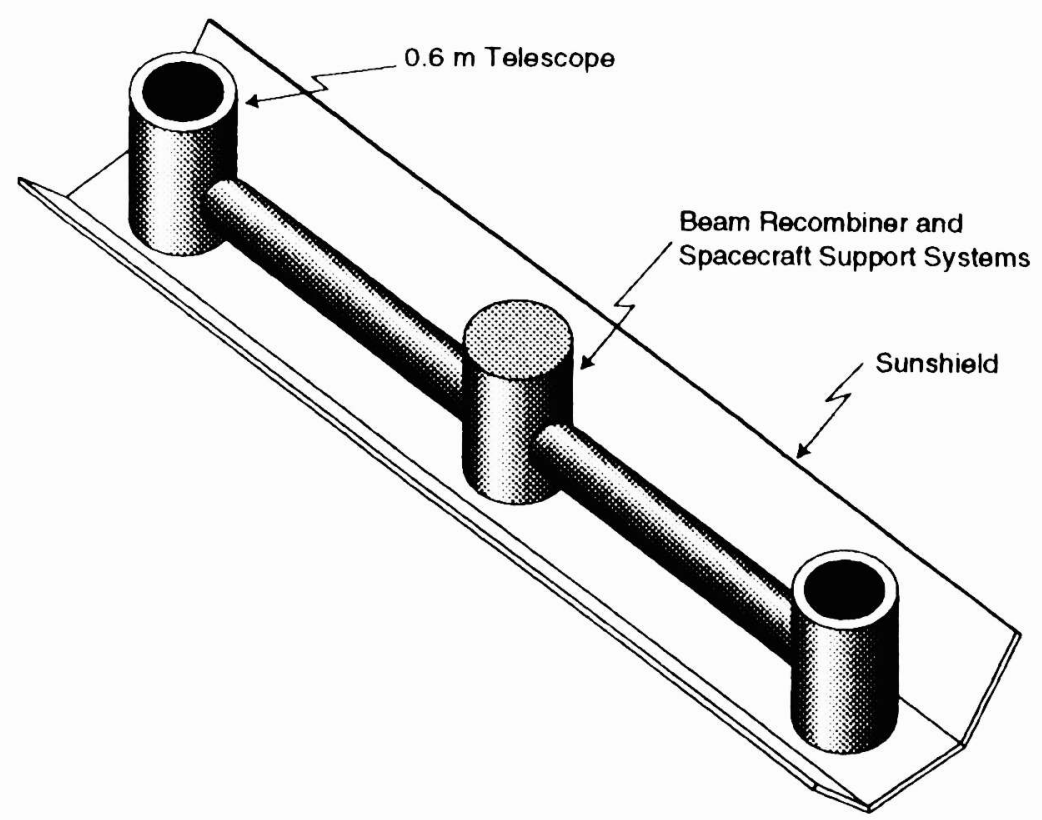

Figure 2. Artist view of the Exo-Zodiacal Mapper.

A simulated observation data set is obtained by applying this response function to a given target composed of a star of a particular spectral type and distance, a surrounding zodiacal disk of specific inclination and two dimensional intensity characteristics, and the background due to our own zodiacal light. Background from the thermal emission of the telescope mirrors is also included. Leakage from the star, due to i) the finite size of the stellar disk compared to the interferometer response map, ii) pointing jitter and ii) imperfect nulling (optical path difference errors, optical alignment errors, figure errors, dust on optical surfaces), is also taken into account. The resulting simulated photo-electron count at the detector (assuming a given optics transmission, detector quantum efficiency, and Poisson distribution photon noise statistics), is determined for each wavelength bandpass and binned for each interferometer rotation angle. A typical simulated data set obtained with a two- 0.6 meter aperture, 10 meter baseline, at 3 AU, for a G2 star at 20 parsecs surrounded by solar system like zodiacal disk inclined at $60^{\circ}$ from the line of sight is tabulated in Table 1 and plotted in Fig. 4.

Table 1. Photons/s at $10 \mu$ mfrom a G-2 star at 20 pc (30\% bandpass)

\begin{tabular}{ccc}
\hline Source & incoming & at detector \\
\hline Solar system zodiacal foreground & 477 & 57 \\
Exo-zodiacal disk & 101 & 1.5 \\
Optics & 2 & 0.5 \\
Star cancellation & $1.410^{6}$ & 26 \\
Star leakage & $1.410^{6}$ & 75 \\
Detector & - & 1 \\
\hline
\end{tabular}

The simulated observation is analyzed with a reconstruction algorithm that determines the set of target disk parameters (dust density and temperature power law exponents $m$ and $n$, the disk inclination $\theta$, and the intensity scaling factor $A$ ). This is done by minmizing the root mean square of the difference between the simulated observational data and a trial model over each spectral band. The minimization algorithm we have used is based 

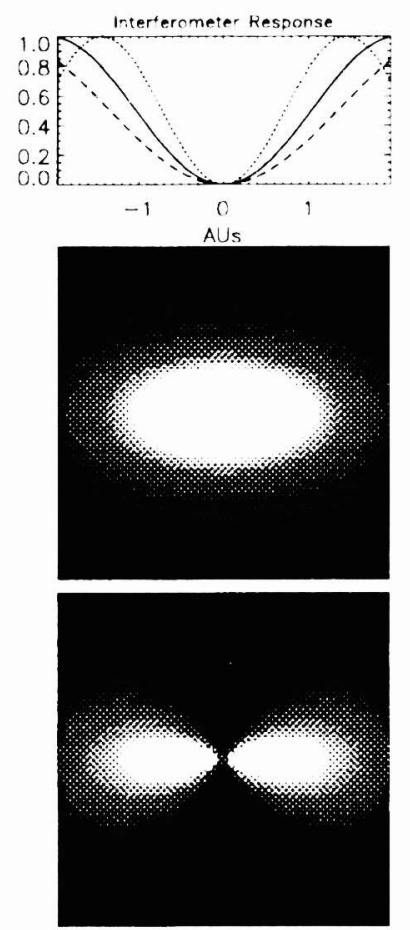

Figure 3. At the top, response function of the nulling interferometer in the direction parallel to its baseline at 7 , 10 and 13 microns (dotted. solid and dashed line respectively). Below, a simulated exo-zodiacal disk with an inclination of $60^{\circ}$ (middle) and seen through the response function of the nulling interferometer (bottom). The interferometer system does not form that "image" and is only sensing its integrated flux.

on the Simplex method (Press et al. 1986) restricted to avoid physically meaningless values in the free parameter space. The fitting parameters are independent and the reconstruction is very robust. In the absence of photon noise, reconstruction is essentially perfect with 18 data points (3 wavelengths and 6 interferometric rotation steps).

\subsection{Simulation results}

Simulations runs have shown that a signal to noise ratio (SNR) of 10 per data point is enough to constrain the disk inclination to $\pm 10^{\circ}$, but an SNR of at least 20 is required to obtain a good solution for the power law exponents. Predictably, the influence of the bandpass is most significant for the temperature distribution parameter. A bandpass of $10 \%$ is sufficient to constrain the temperature distribution, but a large bandpass is desirable to minimize the integration time for a given signal to noise ratio.

Based on simulated observations, Fig. 5 shows the integration time for the detection of an exo-zodiacal disk with the same intensity as ours, as a function of baseline and aperture diameter. The simulation was for a bandpass of 7 to 13 microns in three bands, a G2 target star at 20 parsecs, and the spacecraft located at 3 AU. The sharp rise on the left portion of each curve corresponds to the resolution limit of the instrument: when the baseline is too small, most of the disk gets cancelled in the interferometer null, and the integration time rises accordingly. To the right of this limit, the influence of baseline is less important, and the aperture diameter is the main factor. In that regime, our zodiacal light is the limiting background and the integration time varies as the inverse square of the aperture diameter.

To first order, the interferometer aperture diameter is set by the integration time allocated per star (i.e. by how many stars are to be in the mission lifetime), and the baseline selected to permit adequate resolution of the zodiacal disk (corresponding to the knee in the curves of Fig. 6). To determine these parameters, we have calculated 

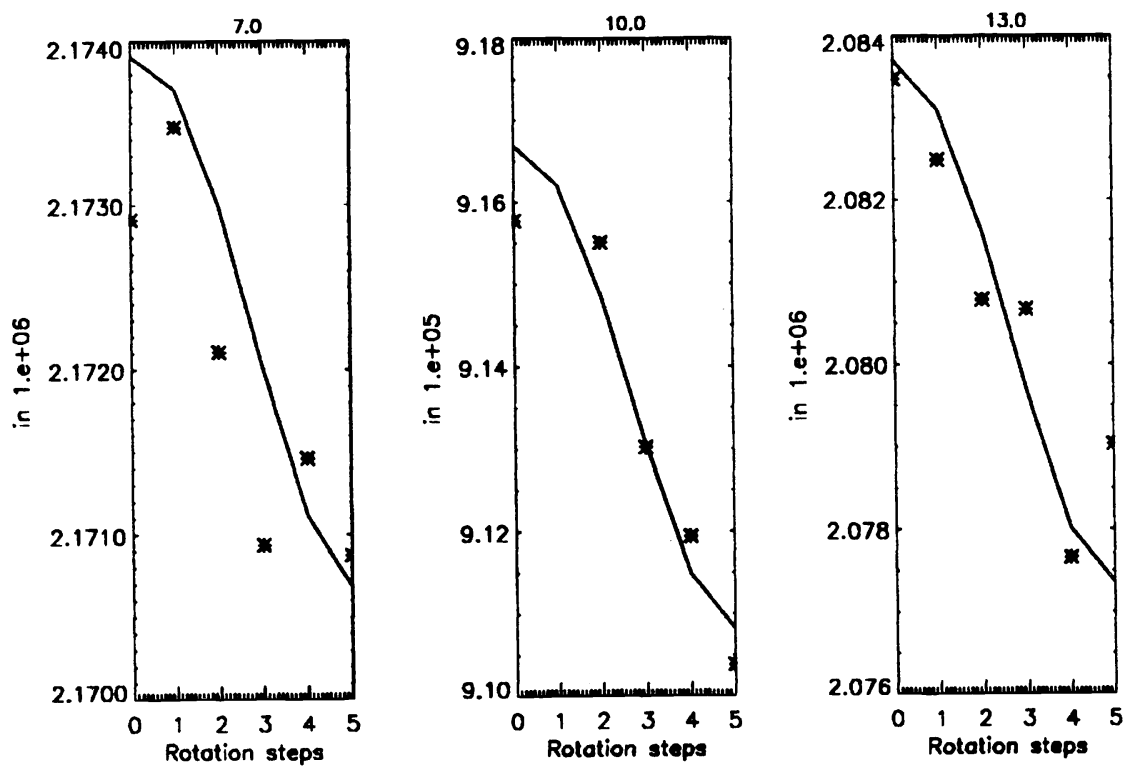

Figure 4. Simulated data set for an exo-zodiacal disk with an inclination of $60^{\circ}$. Each plot shows for each wavelength band the simulated original interferometer output (asteriscs) and the modelized reconstruction as a function of rotation angle over a quarter turn.

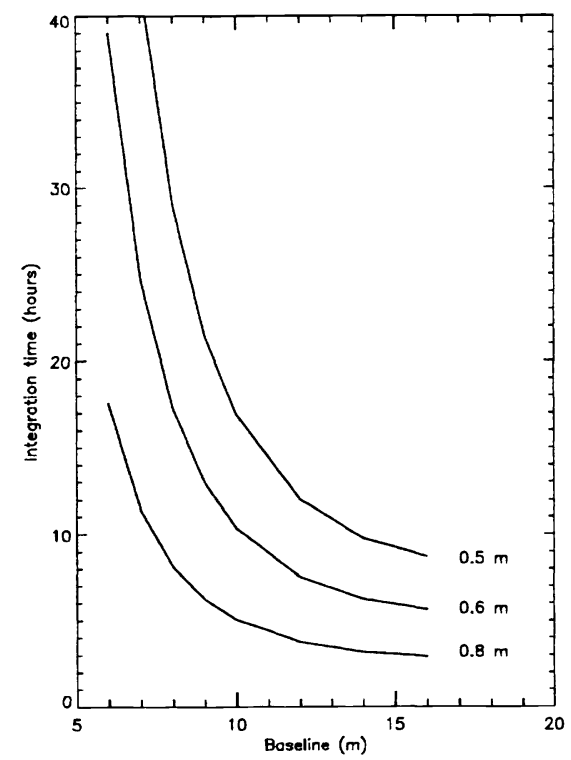

Figure 5. Integration time required to reach a signal to noise ratio of 10 per data point (6 per quarter turn) on an exo-zodiacal disk similar to ours around a G-2 star at 20 parsecs as a function of the baseline and aperture diameter. 


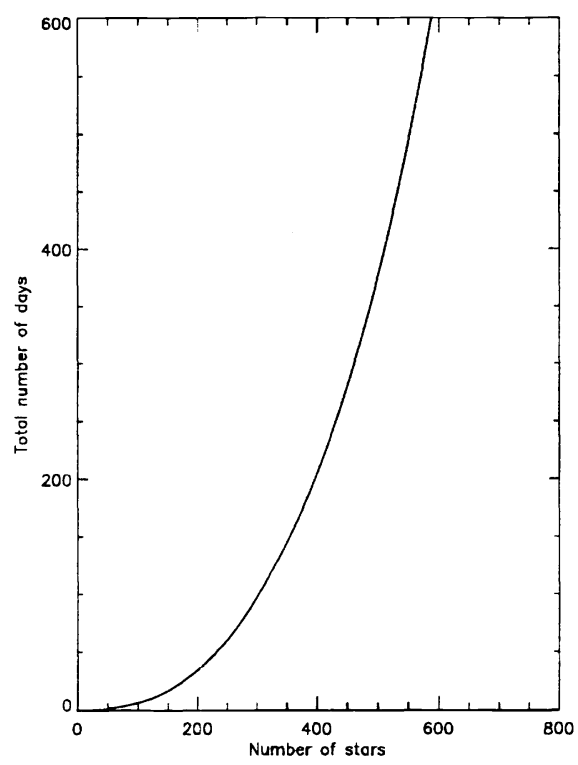

Figure 6. Total number of days required to detect and map exo-zodiacal disks as a function of the desired star sample. The spacecraft is assumed at $3 \mathrm{AU}$, the average signal-to noise ratio per sampling point is 20 and only non binary stars are included. No observation overhead is taken into account.

the exposure time required to detect a zodiacal disk with an intensity similar to ours around each star in the solar neighborhood. We used the data from the Gliese catalog ${ }^{13}$ which extends to 50 parsecs and excluded all known or suspected binary star (since companions are not nulled by the interferometer and would preclude any detection of the much fainter zodiacal disk). The calculation was done assuming an overall transmission of $50 \%$ for the optics, a temperature of 50 Kelvin for the passively cooled telescope mirrors, a coating emissivity of $3 \%$, a quantum efficiency of $65 \%$ for the detector and a signal-to-noise ratio per sample point of 20 . The solar system zodiacal background was calculated for each star based on its ecliptic latitude, but for simplicity, the spacecraft was assumed to be at a constant heliocentric distance of $3 \mathrm{AU}$. Star leakage was included, the star diameter being estimated from its absolute magnitude, spectral class and radiation law, and assuming a line of sight jitter of 1 milliarcsecond. Finally we assumed that the null on axis would not be better than $10^{-4}$ due to residual wavefront errors, and intensity or polarization mismatch.

The results are summarized in Fig. 6. We estimate that a two 0.6-meter aperture, 10-meter baseline interferometer on a $1 \times 4 \mathrm{AU}$ orbit would allow the investigation of about 400 stars over a single pass around the orbit.

\section{OTHER APPLICATIONS OF EZM}

The distinguishing characterisic of the nulling interferometer is its ability to detect faint companions or structures very near a bright point-like source. Although the principal use of the proposed interferometer would be to search for circumstellar disks in the Solar neighborhood, it clearly can be used for other surveys and to make detailed investigations. Examples of other kinds of projects include brown dwarf binary companions, stellar activity of AGB stars, binary companions of AGB Stars, structure of T Tauri Disks, jets of LBV stars, spectrum of QSO and AGN host galaxies and binary searches.

\section{CONCLUSION}

The EZM can solve the important, scientifically well defined problem of the frequency and properties of zodiacal disks around solar type stars through interferometric techniques. Such a well focused problem is precisely what space nulling interferometry needs in order to germinate from concept to reality. Although a simple interferometer, the 
EZM would be a powerful testbed for validating technologies such as deployment of large optical systems, lightweight structures, passive cooling, low noise mid-infrared detectors, ultra-low vibration cryo-coolers, launch to and operation at heliocentric orbit, and of course the critical high precision nulling.

\section{ACKNOWLEDGMENTS}

We thank Robert Brown, Hervey (Pete) Stockman and Rodger Doxsey of the Space Telescope Science Institute and Harley Thronson of NASA Headquarters for their advice and suggestions.

\section{REFERENCES}

1. Beichman, C. A., 1996, A road map for the exploration of neighboring planetary systems, JPL Pub 96-22

2. Mather, J.C., Seery, B.D., Bély, P.Y., 1996, SPIE, 2807, 98

3. Burg R., Petro L,, Bély, P.Y., Gay, J., Rabbia, Y. and Redding, D., 1997, in Proc. Science with the NGST, (in preparation)

4. Bracewell, R., Nature, 1978, 274, 780

5. Bracewell, R., and Mac Phie, R., 1979, Icarus, 38, 136

6. Gay J. and Y. Rabbia, 1996, C.R. Acad. Sci., Paris, 322, serie II b, 265

7. Roddier, F., Roddier, C. and Demarcq, J., 1978, J. Optics, 9, 145.

8. Shao M., 1989, in Proc. NASA/STScI Workshop on the Next Generation Space Telescope, ed P. Bély, C. J. Burrows and G. Illingworth, 160

9. Shao, M., and Colavita, M., 1992, A\&A Reviews, 30, 457

10. Gouy, L.G., 1890, C.R. Acad. Sci., Paris, 110, 1251

11. Born, M, and Wolf, E., 1980, Principles of Optics, Pergamon Press

12. Good, J., 1994, in IRAS Sky Survey Atlas, Appendix G, eds. S.L. Wheelock, T.N. Gautier, J. Chillemi, D. Kester, H. McCallon, C. Oken, J. White, D. Gregorich, F. Boulanger. J. Good, T. Chester

13. Gliese W. and Jahreiss, H., 1989, Preliminary Version of the Third Catalogue of Nearby Stars", (electronic version), Astronomisches Rechen-Institut, Heidelberg 